\title{
Phenomenological Aspects of Grain Boundary Diffusion
}

\author{
I.V. Belova ${ }^{1, a}$ and G.E. Murch ${ }^{1, b}$ \\ ${ }^{1}$ Diffusion in Solids Group, School of Engineering, \\ The University of Newcastle, Callaghan, New South Wales 2308, Australia \\ arina.Belova@newcastle.edu.au, braeme.Murch@newcastle.edu.au
}

Keywords: Grain boundary diffusion, segregation, Monte Carlo methods.

\begin{abstract}
In this paper, the authors review recent progress that has been made in understanding various aspects of grain boundary diffusion at the phenomenological level. We show how the mapping of phenomenological grain boundary diffusion problems onto a lattice readily permits lattice-based random walk theory to be used to address the problem, invariably by Monte Carlo computer simulation methods. Recent advances in calculating concentration profiles and effective diffusion coefficients are described in detail.
\end{abstract}

\section{Introduction}

Grain boundary diffusion can be considered at two theoretical levels, as indeed can solid state diffusion generally. At one level (the atomistic level), the detailed atomic structure of grain boundaries and the corresponding atomic diffusion of atoms within the grain boundaries are treated; see, for example, the following recent reviews [1,2]. At the second level (the phenomenological level), the grain boundaries are considered as connected regions of the material that have a higher (usually single valued) diffusivity than the grains. The phenomenological description enables the measurement and separation (as appropriate) of grain boundary diffusion coefficients, bulk or lattice diffusion coefficients, effective diffusion coefficients and segregation factors [1]. In the present paper, recent progress that has been made in the understanding of various phenomenological aspects of grain boundary diffusion is reviewed.

\section{The Lattice Model}

Most of the recent progress about the phenomenological description of grain boundary diffusion has depended, ironically, on lattice models. The lattice model was originally conceived as a very simple model for describing the actual atomistics of grain boundary diffusion [3]. In the model, the grain boundary is represented by a single plane of sites in which the grain boundary diffusion coefficient of the diffusant is associated with a single atomic jump frequency. The rest of the lattice represents the grains and has an atomic jump frequency associated with the bulk diffusion coefficient of the diffusant. When the jump frequency to and from the grain boundary is different from the other jump frequencies then segregation of the diffusant to the grain boundary can be formally described. It was later recognized that when the jump distance in the lattice is rescaled to any distance and the spatial distribution of the diffusion coefficients (of any number) is arbitrarily chosen, in principle, the basic lattice model can be used to address any phenomenologically conceived diffusion problem. The randomly walking particles that then diffuse on the lattice no longer represent individual atoms but simply are a way of sampling various regions having different diffusivities.

The lattice model is very well suited for addressing phenomenological grain boundary diffusion problems. The Monte Carlo method has invariably been used to deal with the lattice model. Although not strictly a lattice calculation, the earliest Monte Carlo calculation addressing a phenomenological grain boundary diffusion problem was by Brandt [4] who used a floating random walk method. The first formal lattice model calculation was by Murch [5] though the basic idea is a relatively obvious one and has in fact been initiated quite independently on several occasions. The 
basic idea of the method proposed, which has been used with numerous extensions and variants in many subsequent calculations, is as follows. Some $10^{6}$ or more particles are released one at a time into a simple cubic lattice. There are no diffusion correlation effects here since the particles diffuse quite independently. They can be released one by one, or all at once provided multiple occupancy of a site is allowed. Depending on the context of the problem being addressed, the release of particles can be from a source such as a specific point, line or plane or anywhere in the lattice. Segregation in the problem may require a weighted release depending on the location. Each particle randomly explores the lattice for some time $t$ during which time they jump from site to site according to a specified local jump frequency (strictly a probability for that time step). The various jump frequencies multiplied by the jump distance squared are scaled to the largest diffusion coefficient in the system. The highest jump probability is set equal to unity.

In the simplest algorithm, each attempt to jump by a particle advances the 'clock' by one time step. The very large difference between the lattice and grain boundary diffusion coefficients, often four or five orders of magnitude, means that the simplest algorithm is quite inefficient. Various straightforward residence time algorithms have been proposed can increase the efficiency though there is inevitably some extra computational overhead. The jump distance or mesh size can be adjusted to increase efficiency and/or accuracy. For example, when the diffusivity is unchanging over a large region then the mesh size can be increased in that region but where the diffusivity changes rapidly in a region the mesh size can be decreased in order to capture functional detail. A somewhat similar result can be obtained by changing the time increment on the clock according to the position of the particle but this requires somewhat more computational overhead.

Principal outputs of these calculations are frequently the concentration (depth) profiles and depth iso-concentration contour maps away from the source. These concentration profiles are built up simply by determining the number of particles that have reached a given distance from the particle source after time $t$. The (scaled) effective diffusivity $D_{\text {eff }}$ of a material can also be calculated in the long-time limit simply from the Einstein Equation:

$$
D_{\text {eff }}=<R^{2}>/ 6 t
$$

where $R$ is the displacement of a particle in time $t$. Eq. 1 would normally require that the particles are created at random positions in the lattice, but such positions would need to be appropriately weighted if there is segregation in the problem. In general, the time required to calculate the longtime limit effective diffusivity will be directly related to the diffusion length $\left(6 \mathrm{D}_{\text {eff }} \mathrm{t}\right)^{1 / 2}$ : this length needs to be sufficiently large, typically half of the cell size for a system with periodic boundaries.

As an alternative to Eq. 1, a steady state method using Fick's First Law:

$$
J=-D_{\text {eff }} \partial C / \partial y
$$

is also possible when a source and a sink of particles are introduced. Then an effective particle concentration gradient $\partial C / \partial y(C$ is the concentration of the particles and $y$ is the distance normal to the source plane) can be obtained by creating a particle at the source and annihilating it at the sink. The apparent flux $J$ of particles between two neighbouring planes between source and sink can be readily calculated over a long time period $t[6]$.

Overall, these programs are very straightforward to write and can be readily adapted to quite complex geometries and boundary conditions. They represent a valuable alternative to finite difference and finite element methods for addressing and solving complex phenomenological diffusion problems [7]. 


\section{Dislocation Pipe Tracer Concentration Depth Profiles}

The phenomenological problem of diffusion in the presence of dislocation pipes has been examined only occasionally using the lattice model. The isolated dislocation pipes can be simply represented by lines or clusters of lines normal to the surface [5]. In the first study, tracer concentration depth profiles from a thin-film source were determined and the results were found to be in very good agreement with the analytical solution provided by Le Claire and Rabinovitch [8]. A very slight curvature appears in the simulated tail section of the concentration depth profiles when plotted against $y$. Since the curvature can be removed when one imposes the condition that tracers leaving the dislocation pipe never return, this suggests that this is a tacit assumption in the theoretical analysis. In another calculation [9], a study was made to examine the conditions under which the dislocation pipe diffusion coefficient of a diffusant can be determined from a grain boundary type analysis of the tail of the tracer concentration profile. This required permitting dislocation pipes to approach one another until they effectively behave as a grain boundary slab.

\section{Grain Boundary Tracer Concentration Depth Profiles}

The problem of determining tracer concentration depth profiles in the presence of grain boundaries having different geometries has been studied on many occasions. In the first calculation, Brandt [4] was able to verify the essential correctness of the well-known Whipple solution [10] for describing the tracer concentration profiles for diffusion from an infinite tracer source at the surface in the presence of isolated grain boundary 'slabs' normal to the surface. Several important suggestions were made by Brandt in this paper including impurity segregation and the simultaneous simulation of grain boundary, bulk and surface diffusion and these aspects have been examined since by others.

Lavine and Losee [11] examined impurity concentration depth profiles from a thin film source into an array of finite sized grains arranged in a 'brick wall' configuration. Segregation of the impurity to the grain boundary was allowed for. They found secondary peaks at locations where the grain boundaries are parallel to the surface.

Murch and Rothman [13] examined the behavior of the tracer concentration depth profiles from a thin film source when the diffusion fields from equally spaced parallel grain boundary slabs are permitted to overlap extensively: this is also frequently called the Harrison type-A kinetics regime [14]. The calculation concentrated on the problem of determining the limits of the well-known Hart Equation [15] for the effective diffusion coefficient $D_{\text {eff }}$ as determined from the concentration depth profiles for diffusion from a thin film source in the presence of grain boundary slabs normal to the surface:

$$
D_{\text {eff }}=g D_{\mathrm{gb}}+(1-g) D_{1}
$$

where $g$ is the fraction of sites in the grain boundary, $D_{1}$ is the diffusion coefficient in the lattice and $D_{\mathrm{gb}}$ is the grain boundary diffusivity. Because of computational demands this early calculation was of limited scope. When much superior computational resources became available, the problem was revisited later in much greater depth by Belova and Murch [16]. In the later study, it was shown that the upper limit for Harrison type-A kinetics regime occurs when $\Lambda\left(=L /(D t)^{1 / 2}\right)$ is approximately equal to 0.4 and this is essentially independent of the $\beta$, sometimes called the 'Le Claire parameter', $\left(\beta=\delta D_{\mathrm{gb}} / 2 D_{\mathrm{l}}\left(D_{\mathrm{l}} t\right)^{1 / 2}\right)$. These workers found a small transition region between 0.4 and 0.2 , which is somewhat dependent on $\beta$ before the Harrison type-B kinetics regime is formally entered. When a condition for fast surface diffusion is introduced, the upper limit for Harrison type-A diffusion kinetics is pushed up slightly to 0.7 . These upper limits are much higher than the value of 0.0033 originally estimated by Harrison for spherical grains [14]. It is clear that a tracer atom need not sample a large number of grains and grain boundaries in order for its diffusion 
coefficient to be given by the Hart Equation (Eq. 3). In fact, this result implies that the diffusion length need only be roughly the spacing between the (parallel) grain boundary slabs. This result is in fact similar to what was found for dislocation pipe diffusion where the upper limit for Harrison type-A diffusion kinetics is known from a near-exact analysis to occur at $\Lambda=0.1$ [8].

Divinski et al. [17] examined more closely the transition from the Harrison type-A kinetics regime to the type B kinetics regime and focused especially on the intermediate region. They found that the concentration depth profiles (from a thin film source) are well linear when plotted against $y^{2}$ (i.e. Gaussian) for $\Lambda \leq 0.3$. At larger values of $\Lambda$ the profiles become curved and only a crude estimate of $D_{\text {eff }}$ is possible although this can be improved if only the first few points are used. They showed that the Harrison type-B kinetics regime is probably not really well established until $\Lambda$ is approximately equal to 4 . Between $0.3 \leq \Lambda \leq 4$ they identified what they believe to be an intermediate region, formally called the 'AB transition regime', where the profiles show a pronounced negative curvature in a $y^{6 / 5}$ plot. Divinski and Larikov [18] had earlier provided an analysis in this region wherein the depth profiles can be plotted against $y^{3 / 2}$ and the grain boundary diffusion coefficient can be extracted provided the grain boundary width $\delta$ is known or estimated:

$$
D_{g b} \approx \frac{16.65 D_{\ell}^{0.1}}{(1+\delta / d) \delta^{0.2} t^{0.9}}\left(-\frac{\partial \ln C}{\partial y^{3 / 2}}\right)^{-4 / 3}
$$

Hodge [19] has performed a number of calculations of concentration profiles from a thin film source where the grains are randomly oriented. He used an f.c.c. lattice rather than the usual simple cubic lattice because it provides for a larger number of grain boundary orientations for a given mesh size. The effect of grain size and distribution, grain shape and the ratio $D_{\mathrm{gb}} / D_{1}$ were all considered. Hodge made an extensive exploration of these parameters and attempted to fit the concentration depth profiles $C(y, t)$ with an empirical equation:

$$
C(y, t)=A \exp \left(-y^{\eta} / D_{\text {app }} t\right)
$$

where $A$ is a constant and $D_{\text {app }}$ is an apparent diffusivity and $\eta$ is a function of $D_{\mathrm{l}} / D_{\mathrm{gb}}$.

Metsch et al. [20] examined simultaneous lattice, grain boundary and surface diffusion especially for the case where the rate of surface diffusion is greater than bulk diffusion but still slower than grain boundary diffusion. They explored the effect on the shape of the concentration depth profiles from a thin film tracer source. The possibility of fast surface diffusion was later introduced by Mishin and Razumovskii [21] into the solution of the corresponding diffusion equation for the grain boundary diffusion problem. In the presence of grain boundaries, fast surface diffusion leads to a depletion of tracers near the surface and a local maximum in the tracer concentration depth profile. In effect, the fast surface diffusion permits the 'pumping away' of tracers by the grain boundaries.

Another study [22] described a calculation of the contour angle made by iso-concentration lines with an isolated grain boundary for the case of diffusion from a thin film source. Since there are no relations available for this source condition that involve the contour angle and the grain boundary and lattice diffusion coefficient, two new relations were derived in the spirit of the Fisher [23] and Whipple [10] treatments. Monte Carlo results showed that both relations would overestimate the grain boundary diffusion coefficient by way of a measurement of the contour angle. 


\section{Effective Diffusion Coefficients}

When some tracer atoms sample only the grain boundaries and others sample only the lattice whilst still others sample both during the diffusion anneal, the tracer concentration depth profiles for diffusion from the thin-film source show two distinct sections. The conditions where this occurs are usually referred to as the Harrison type-B kinetics regime. The second or tail section of the depth profile can be processed to extract either the product $\delta D_{\mathrm{gb}}$ where $\delta$ is the apparent grain boundary width, or, if impurity diffusion is being investigated, $s \delta D_{\mathrm{gb}}$ where $s$ is the segregation factor, defined as the ratio of the concentration of solute in the grain boundaries to the concentration of solute in the grains in the case of impurity diffusion. The first section of the concentration depth profile is invariably approximately Gaussian (for diffusion from a thin film source). When the contribution to diffusion from the grain boundaries is relatively minor, this Gaussian region describes only the lattice diffusion coefficient $D_{1}$. As the grain boundary contribution to diffusion grows, the diffusion coefficient measured in this Gaussian region increases until it finally becomes the effective diffusion coefficient $D_{\text {eff }}$ for diffusion of that diffusant in the material and the Harrison type-A kinetics regime is formally entered. (Between these two kinetics regimes there is also an apparent intermediate $\mathrm{AB}$ region as mentioned above.) The effective diffusion coefficient is a composite of the lattice diffusion coefficient and the grain boundary diffusion coefficient of the diffusant. In grain boundary diffusion studies, the effective diffusion coefficient has been invariably expressed using the Hart Equation (expressed in the more general form with the inclusion of the solute segregation factor [1]:

$$
D_{e f f}=\frac{s g D_{g b}+(1-g) D_{\ell}}{1-g+s g}
$$

Note that $s$ equals unity for the case of self diffusion. The Hart Equation is exact for parallel diffusion paths in the diffusion direction, i.e. the grain boundaries are considered only as parallel slabs normal to the surface. The Hart Equation is in fact mathematically analogous to an electrical circuit having parallel electrical conductances.

In real material, however, diffusing tracer atoms will inevitably cross one or more grain boundaries in the diffusion direction during the diffusion anneal thus making the Hart Equation problematic: one has, in effect, the equivalent of an electrical circuit situation having a combination of parallel and series conductances. A number of Monte Carlo calculations have been made in which grains are represented as cubes or as spheres in various configurations with and without segregation [24-26]. These calculations make it very apparent that the modified Maxwell Equation [27] gives a very much better agreement with the Monte Carlo data than the Hart Equation. The modified Maxwell Equation for $D_{\text {eff }}$ is:

$$
D_{e f f}=\frac{s D_{g b}\left((3-2 g) D_{\ell}+2 s g D_{g b}\right)}{(1-g+s g)\left(g D_{\ell}+(3-g) s D_{g b}\right)}
$$

These findings make it quite clear that in general the modified Maxwell Equation should replace the Hart Equation in the interpretation of $D_{\text {eff }}$ in real materials. One can expect that with the Maxwell Equation the transition from Harrison type-B kinetics regime to the type-A kinetics regime will occur at somewhat different values of $\Lambda$ than given above for the case of parallel boundary slabs and that the intermediate $\mathrm{AB}$ regime may also change too, but these matters have yet to be investigated for even simple grain models. The actual numerical differences between the Maxwell and Hart Equations are of course of interest for typical values of the lattice and grain boundary diffusion coefficients and the segregation factor. In Fig. 1 we have plotted the ratio of $D_{\text {eff }}$ (as obtained from the Hart Equation) to $D_{\text {eff }}$ (as obtained from the Maxwell Equation) as a function of $g$ for values of $s D_{\mathrm{gb}} / D_{1}$ ranging from $10^{3}$ to $10^{8}$. It is seen that even for large grained microcrystalline 
material where $g \sim 3 \delta / d$ (where $d$ is the grain diameter) may be as low as $3 \times 10^{-5}$ with no segregation, the use of the Hart Equation would undoubtedly overestimate $D_{\text {eff }}$ by at least $10 \%$ if $D_{\mathrm{gb}} / D_{\mathrm{l}}=10^{4}$. This overestimate would rapidly increase to $50 \%$, but not more, for higher values of $g$ or higher values of $D_{\mathrm{gb}} / D_{\mathrm{l}}$. The influence of $s$ is simply to push the same overestimate of $D_{\text {eff }}$ to lower values of $g$. For nanocrystalline materials, where $g$ can of course be much higher, perhaps of the order of 0.1 , the use of the Hart Equation would generally overestimate $D_{\text {eff }}$ by about $45 \%$ and this is essentially independent of the degree of segregation. However, it should be noted that in nanocrystalline materials there is some evidence that the effective diffusion coefficient may have further high mobility contributions [17].

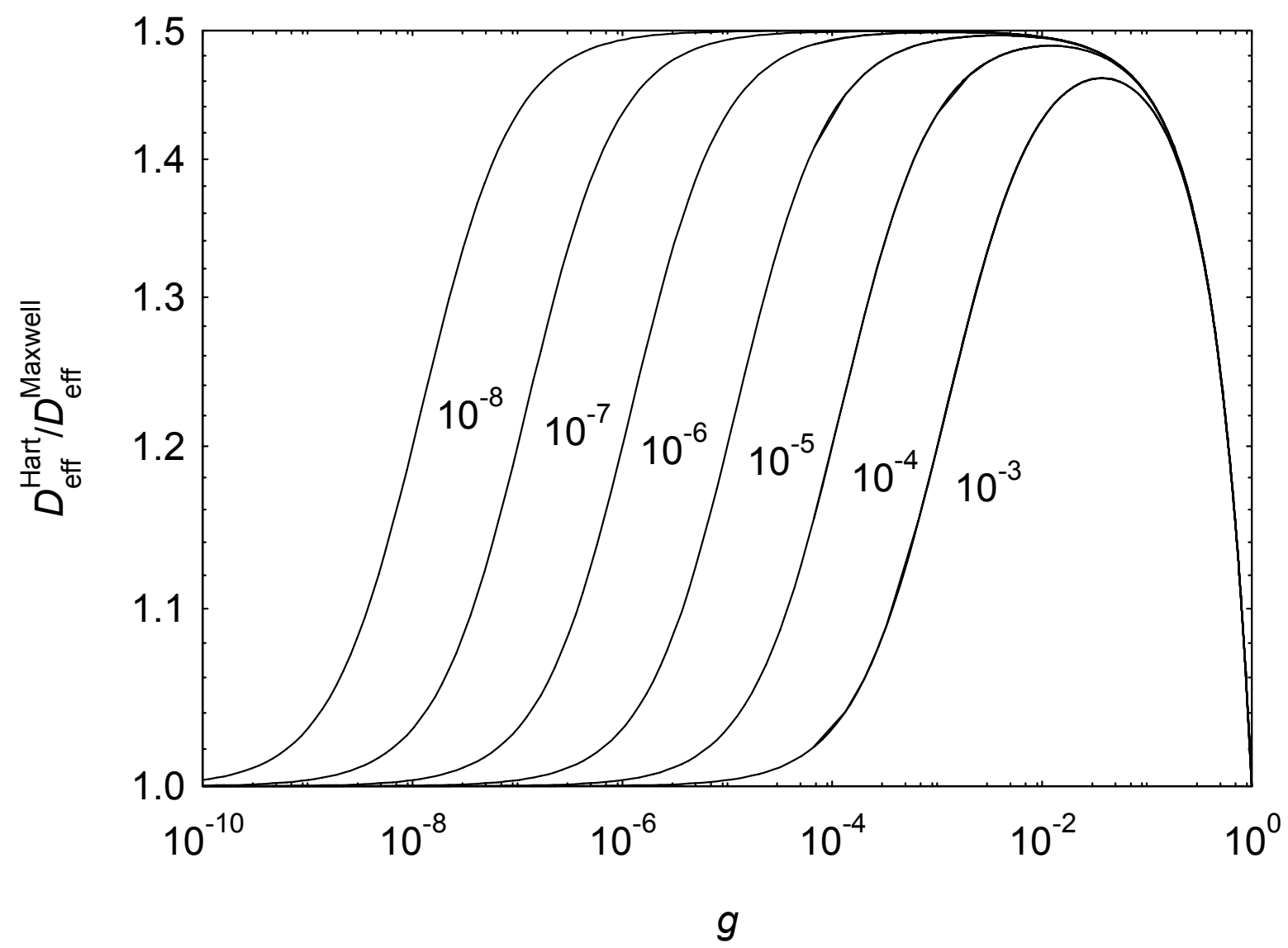

Figure 1. Ratio of $D_{\text {eff }}$ (as obtained from the Hart Equation) to $D_{\text {eff }}$ (as obtained from the Maxwell Equation) as a function of $g$ for values of $D_{1} / s D_{\mathrm{gb}}$ ranging from $10^{-3}$ to $10^{-8}$ (lines are marked with corresponding numbers).

Using the lattice model and Monte Carlo methods, Belova and Murch [28] calculated $D_{\text {eff }}$ in stable two-phase material using a cubic grain model. The interphase boundaries were treated as high mobility paths in the same way as regular grain boundaries. They derived several expressions for $D_{\text {eff }}$ along the lines of the Maxwell Equation and obtained much better agreement than with the equivalent of the Hart Equation. The latter would be appropriate if the interphase boundaries were normal to the tracer source. The time dependence of concentration depth profiles for this situation has not yet been explored.

At short diffusion times, in both microcrystalline and nanocrystalline material, solute coming from a grain boundary source cannot sample enough of the grains for an equilibrium partitioning of the solute to be properly established. Put another way, if the diffusion length is very much less than the size of the grains, it is unlikely that an equilibrium segregation of the solute (as reflected in the segregation factor) between the grains and grain boundaries is possible, though this is very frequently the tacit assumption. This problem was addressed by assuming local regions (delineated by the diffusion length) in the region of the grains adjacent to the grain boundary where the solute is properly equilibrated. Belova and Murch [29] derived expressions based on the Hart and Maxwell 
Equations for an apparent time-dependence of the effective solute diffusion coefficient. Monte Carlo calculations using the lattice model show that these expressions work quite well for solute diffusion parallel to the grain boundary and perpendicular to the grain boundary (but in the latter case only for the weak segregation case).

\section{Summary}

In this paper, recent progress that has been made in understanding various aspects of grain boundary diffusion at the phenomenological level has been reviewed. We showed how the mapping of phenomenological grain boundary diffusion problems onto a lattice readily permits lattice-based random walk theory to be used to address the problem, usually by Monte Carlo computer simulation methods. Recent advances in the calculation of concentration profiles and effective diffusion coefficients were described in detail.

\section{Acknowledgements}

We wish to thank the Australian Research Council for its support of this work under the Discovery Project Grants Scheme. One of us (IVB) wishes to thank the Australian Research Council for the award of a Professorial Fellowship.

\section{References}

[1] I. Kaur, Y. Mishin and W. Gust, Fundamentals of Grain and Interphase Boundary Diffusion (Wiley, Chichester 1995).

[2] Y. Mishin, Defect and Diffusion Forum Vol. 194-199 (2001), p.1113.

[3] P. Benoist and G. Martin, Thin Solid Films Vol 25 (1975),p.181.

[4] W.W. Brandt, J. Chem. Phys. Vol. 59 (1973), p.5562.

[5] G.E. Murch, Diffusion and Defect Data Vol. 32, (1983), p.1.

[6] I.V. Belova and G.E. Murch, unpublished work.

[7] T. Fiedler, A. Öchsner, N. Muthubandara, I.V. Belova and G.E. Murch, Materials Science Forum, to be published.

[8] A.D. Le Claire and A. Rabinovitch, in Diffusion in Crystalline Solids, edited by G.E. Murch and A.S. Nowick (Academic Press, Orlando 1984).

[9] I.V. Belova and G.E. Murch, Proc. Mat. Res. Soc. Vol. 677 (2001), p. AA7.7 (electronically published).

[10] R.P.T. Whipple, Philos. Mag. Vol. 45 (1954), p.1225.

[11] J.P Lavine and D.L. Losee, J. Appl. Phys. Vol. 59 (1984), p.924.

[12] J.P. Lavine, in Simulation of Semiconductor Devices and Processes, edited by K. Board and D.R.J. Owen (Pineridge Press 1984).

[13] G.E. Murch and S.J. Rothman, Diffusion and Defect Data Vol. 42 (1985), p.17.

[14] L.G. Harrison, Trans. Farad. Soc. Vol. 57 (1961), p.1191.

[15] E.W. Hart, Acta Metall. Vol. 5 (1957), p.597.

[16] I.V. Belova and G.E. Murch, Philos. Mag. Vol. 81 (2001), p.2447.

[17] S.V. Divinski, F. Hisker, Y.-S. Kang, J.-S. Lee and Chr. Herzig., Zeit. Metallk. Vol. 93 (2002), p. 256.

[18] S.V. Divinski and L.N. Larikov, Defect and Diffusion Forum, Vol. 143-147 (1997), p.1469.

[19] J.D. Hodge, J. Am. Ceram. Soc. Vol. 74 (1991), p.823.

[20] P. Metsch, F.H.M. Spit and H. Bakker, Phys. Stat. Sol. (a) Vol. 93 (1986), p.543.

[21] Y. Mishin and I.M. Razumovskii, Acta Metall. Mater. Vol. 40 (1992), p.597.

[22] I.V. Belova and G.E. Murch, Proc. Mat. Res. Soc. Vol. 677 (2001), p.AA7.20 (electronically published).

[23] J.C. Fisher, J. Appl. Phys. Vol. 22 (1951), p.74. 
[24] I.V. Belova and G.E. Murch, J. Phys. Chem. Solids Vol 64 (2003), p.873.

[25] I.V. Belova and G.E. Murch, Mass and Charge Transport in Inorganic Materials (Techna, Faenza 2003), p. 225.

[26] I.V. Belova and G.E. Murch Proc. Mat. Res. Soc. Vol. 731 (2002), p.W5.4 (electronically published).

[27] J.R. Kalnin, E.A. Kotomin and J. Maier, J. Phys. Chem. Solids Vol. 63 (2002), p.449.

[28] I.V. Belova and G.E. Murch, Philos. Mag. Vol. 84 (2004), p.17.

[29] I.V. Belova and G.E. Murch, Proc. Mat. Res. Soc. Vol. 731 (2002), p.W5.4.1 (electronically published). 
Diffusion in Solids and Liquids

doi:10.4028/3-908451-36-1

Phenomenological Aspects of Grain Boundary Diffusion

doi:10.4028/3-908451-36-1.483 